\title{
Study of multibeam synthetic aperture interferometric imaging algorithm
}

\author{
Wei Sun ${ }^{1,2, a}$, Tian Zhou ${ }^{1,2, b}$, Xiaojing Wang ${ }^{1,2, c}$, Bo Wei ${ }^{1,2, d}$, Haisen $\mathrm{Li}^{1,2, \mathrm{e}}$ \\ ${ }^{1}$ Acoustic Science and Technology Laboratory, Harbin Engineering University, Harbin 150001, \\ China; \\ ${ }^{2}$ College of Underwater Acoustic Engineering, Harbin Engineering University, Harbin 150001, \\ China \\ ehsenli@126.com
}

Keywords: multibeam synthetic aperture sonar; interferometric; imaging algorithm

\begin{abstract}
Based on the model of multibeam synthetic aperture sonar (MBSAS) and the principle of multiple subarray interferometic, a high resolution multibeam synthetic aperture interferometric imaging algorithm was proposed. Firstly, the algorithm got the target rough position via the Back-Projection (BP) algorithm of single array element and the multiple beamforming of the whole array. Then the multiple subarrays interferometry was adopted to get the more accurate Direction Of Arrival (DOA). The algorithm solved the problem that the imaging resolution was limited by the number of beam in the MBSAS BP imaging algorithm. The simulation shows this new method can obtain the more accurate target position and higher resolution images comparing to the traditional MBSAS BP imaging algorithm.
\end{abstract}

\section{Introduction}

Synthetic Aperture Sonar (SAS) is currently a hot research topic in the field of marine exploration, and is mainly used in the detection of seabed topography and underwater targets ${ }^{[1-4]}$. The traditional SAS is a two-dimensional high-resolution imaging sonar, while the multibeam synthetic aperture sonar is the development of SAS, which can get the orientation of the target and give a three-dimensional image by using a linear receiving array ${ }^{[5]}$.

In the system of MBSAS, the received signal of each receiving array element is processed with the synthetic aperture algorithm, which can determine the azimuth position of the target. Then the beamforming of the synthesized signals was made to get the DOA and time of arrival (TOA). Therefore, we can use the azimuth, DOA and TOA to calculate the three-dimensional coordinate of the target. But this MBSAS imaging method can only estimate the sound intensity values in the spindle of the beam. And this discrete sampling measuring decreases the imaging resolution.

MBSAS combines SAS and multibeam bathymetric sonar (MBBS), so the theory of MBBS can be used in the MBSAS. In the system of MBBS, the multibeam coherent principle can solve the problem that the resolution is affected by the number of beams. This method can obtain high bathymetry precision without increasing the number of beam and the cost of hardware ${ }^{[6]}$. Therefore, the MBSAS imaging algorithm and multibeam coherent principle are combined together to obtain high resolution in paper.

\section{Theory analysis}

In the system of MBSAS, the receiving array is a linear array constituted with non-directional array element. During operation, the sonar make uniform linear motion and the transmitting signal is CW. The sonar moves along $\mathrm{y}$-axis direction and the receiving array is along the $\mathrm{x}$-axis. The transmitting array locate at $(0, v t, 0)$ and the location of target is $\left(x_{T}, y_{T}, z_{T}\right)$.

As a single element, the most direct imaging approach is the Back-Projection (BP) algorithm. For each pixel of the swath, calculating and summing the contribution of all echoes, which can get 
each array element y-r image. If the echo data of the No.n element in the No.p ping is $S_{0}(n, p, t)$, the pixel value of the point $(y, r)$ in the swath is

$$
S_{1}(n, y, r)=\sum_{p} S_{0}(n, p, t) e^{j 2 \pi f_{0} \tau(y, r)}=\operatorname{rect}\left(\frac{\frac{r-r_{T}}{c}-\frac{\delta}{2}}{\delta}\right) \operatorname{sinc}\left(\frac{2 f_{0}\left(y-y_{T}\right) v T}{c \sqrt{\left(y-y_{T}\right)^{2}+r^{2}}}\right) e^{j \frac{4 \pi f_{0}}{c}\left(r-r_{T}\right)}
$$

Where $\delta$ is the Pulse width, $\mathrm{T}$ is the synthetic aperture time.

We can get high azimuth resolution image after the echo data is processed by the synthetic aperture algorithm. Then we should do horizontal beamforming with the processed data on the basis of phase information for the target angle information. Assuming that the target is in the far field, the beam angle control in $\theta_{i}$ in the azimuth position $y$, each array element should compensate for the phase difference $\varphi_{i}=2 \pi d \sin \theta_{i} / \lambda$, where $d$ is spacing between the receiving array element. The beamforming result is

$$
S_{2}\left(\theta_{i}, y, r\right)=\sum_{n} S_{1}(n, y, r) e^{j n \varphi_{i}}
$$

It can get the $y-r-\theta$ image after the synthetic aperture of each array element and horizontal beamforming are done, but the value is only on the beam axis. In order to get the target intensity value within the beam, we have to adopt the multi subarray interferometric technology. It is accomplished by dividing the received transducers into the two same number of subarrays, then the beamforming is done with the data processed by the synthetic aperture method. Finally, we can get the accurate DOA of the target according to the phase difference of the same beam. The linear array is divided into two groups, each one has $M$ elements and the two subarrays center spacing is $D=(K-M) d$, assume the signal reflected by the target incident to the receiving transducer array at an angle $\theta_{\text {ttar }}$ from the far-field, the phase difference of the same beam in the two subarrays is

$$
\Delta \varphi=(K-M)\left(\varphi-\varphi_{i}\right)=2 \pi D\left(\sin \theta_{t t a r}-\sin \theta_{i}\right) / \lambda
$$

By using threshold detection method to determine the slant range $R$, azimuth $y_{\text {tar }}$ and the beam $\theta_{\text {tar }}$ of the target on the basis of the MBSAS BP $y-r-\theta$ image. Then the multiple subarrays beamforming in the beam $\theta_{\text {tar }}$ is done on the azimuth position $y_{\text {tar }}$ with the data processed by the synthetic aperture method. After that we adopt multiple subarrays coherent principle to obtain the accurate DOA $\theta_{\text {ttar }}$ of the target. Finally the three-dimensional space coordinates of the target can be calculated by $x_{\text {tar }}=R \sin \theta_{\text {ttar }}, z_{t a r}=R \cos \theta_{\text {ttar }}$.

\section{Simulation}

In order to verify the effectiveness of the algorithm proposed in this paper. Firstly, multiple points targets were imaged by the MBSAS BP and MBSAS interferometric imaging algorithm. Then the underwater 3-D target was imaged by the MBSAS interferometric imaging algorithm. The simulation parameters are shown in Table 1. 
Table 1. The simulation parameters

\begin{tabular}{cccc}
\hline parameter & value & parameter & value \\
\hline Signal frequency & $30[\mathrm{kHz}]$ & Element spacing of received array & $0.024[\mathrm{~m}]$ \\
Pulse width & $50[\mathrm{us}]$ & The sampling rate & $100[\mathrm{kHz}]$ \\
Acoustic velocity & $1480[\mathrm{~m} / \mathrm{s}]$ & The speed of & $1[\mathrm{~m} / \mathrm{s}]$ \\
Pulse repetition interval & $0.1[\mathrm{~s}]$ & The number of received array element & 31 \\
\hline
\end{tabular}

The comparison between the MBSAS BP and MBSAS interferometric imaging algorithm In the simulation, 16 points were processed by the MBSAS BP and MBSAS interferometric imaging algorithm. The received beam width is $60^{\circ}$, from $0^{\circ}$ to $60^{\circ}$, and the beam spacing is $2^{\circ}$. Fig. 3 show the results, where the - represents the real position, the + represents the position calculated by the traditional BP imaging algorithm and the o represents the position calculated by the new interferometric imaging algorithm.

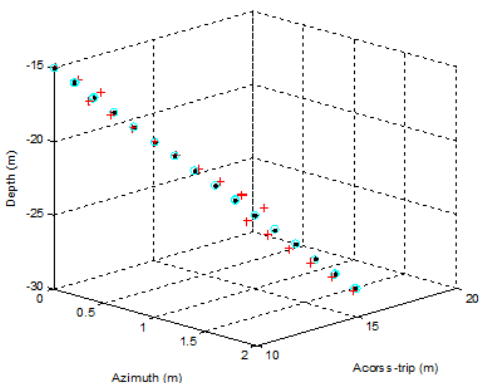

(a)

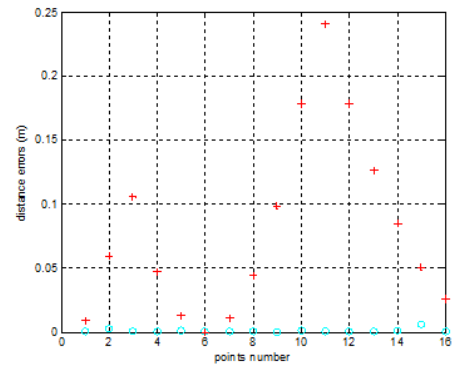

(b)

Figure 3. The simulation results of point targets. (a) The calculated targets position; (b) The distance error

\section{The 3-D target simulation results}

Fig. 4 is MBSAS interferometric imaging results of underwater target. For the 3-D target, the shadow is also an important parameter to determine the target information, thus taking into account the acoustic shadow zone in the simulation. The target is a rectangular and the cube size is $0.8 \mathrm{~m} * 2 \mathrm{~m} * 2 \mathrm{~m}$, where the depth of the target is $8-10 \mathrm{~m}$, the along-track location is $-0.4-0.4 \mathrm{~m}$, the across-trip position is 4-6m. In constructing the goals and seabed echo, using the points in the target surface indicate the target and using binning method to calculate the shaded area, besides assuming the seabed is flat. Fig.4 (a) show the location coordinates of the calculated target and the seabed, it can be seen the shadow is very clear and the target location is very precise. Fig.4 (b) shows the interpolated result.

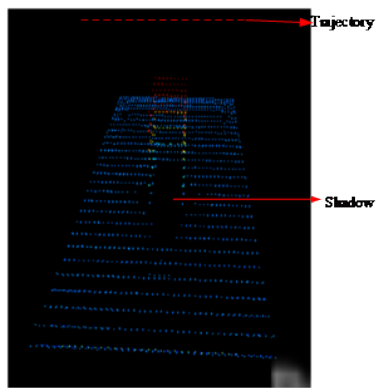

(a)

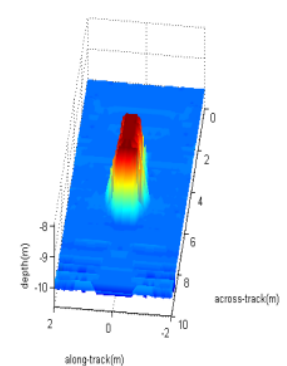

(b)

Figure 4. The imaging results of underwater target. (a) The MBSAS interferometric imaging results; (b) The interpolated result 


\section{Conclusion}

Multibeam synthetic aperture sonar is a full coverage and three-dimensional imaging sonar on the basis of the synthetic aperture sonar, but its depth accuracy is limited by the number of beams in the traditional MBSAS imaging methods. A new multibeam synthetic aperture interferometric algorithm is proposed to solve this problem. We can see that this method can obtain the target angle within the beams, therefore the depth accuracy is improved and the measured target position is more exact contrast with the traditional MBSAS imaging algorithm. For the 3-D target imaging results of the MBSAS interferometric imaging algorithm, both the shape and the shadow of the target have high resolution, which further illustrate the superiority of the new algorithm.

\section{Acknowledgment}

Research in this paper was supported by Natural Science Foundation of China (Grant No.41376103).

\section{References}

[1] CHEN Qiang, TIAN Jie, et al. Speckle suppression for Synthetic Aperture Sonar imaging based on multiple resolution analysis[J]. Applied Acoustics.2012, 31(4): 262-266.

[2] A J Hunter, M P Hayes. Towards more accurate shadow modeling for simulated SAS imagery. [C]// IEEE Oceans-Europe. USA: IEEE, 2005: 713-718.

[3] HAYES P M, GOUGH T P. Synthetic Aperture Sonar: A Review of Current SAS Status[J]. IEEE Journal of Oceanic Engineering.2009,.34(3):207-224

[4] Li Haisen, Xu, Jian, Zhou, Tian.Underwater acoustic optical imaging system[C].International Conference on Signal Processing Proceedings. Beijing, China. 2010:p422-425

[5] Yonghong Yao, Tian Zhou, Haisen Li, et al. Research on the dot-by-dot imaging algorithm for multi-beam SAS based on a new array configuration[C]. 10th International Conference on Signal Processing. Beijing, China. 2010:p2369-2372

[6] XU chao, LI Haisen et al. Multibeam interferometric seafloor imaging technology[J]. Journal of Harbin Engineering University.2013, 34(9): 1159-1164 